\title{
Septic venous thrombosis as an unexpected complication of acute suppurative otitis media. case
} report

\begin{abstract}
Cerebral venous thrombosis is a rare condition that primarily affects women. It has been described in association with local factors (meningitis, sinusitis, cellulite and tumors) and systemic factors such as thrombophilia and other blood disorders. The most common site is the lateral sinus, followed by the sagittal sinus. In $30-40 \%$ of cases it affects more than a venous sinus. The most common clinical manifestation is headache. We present the case of a 65-year-old male patient with a history of ethanolism and smoking, admitted to the Emergency Department for a four-day follow-up, fever, dyspnea and temporary disorientation that worsened in the last 24 hours with an altered state of consciousness. Evidenced by diagnostic computed tomography (CT) a ventriculitis and a defect in the filling of the transverse sinus and left sigmoid in apparent relationship with thrombosis; and opacification of the middle ear by inflammatory process / otitis media. Thrombosis of cerebral venous sines (CVT) is considered difficult to diagnose due to the wide variety of signs and symptoms that can simulate a large number of other entities, it is important to have this diagnosis always present, and it is essential that after the diagnostic suspicion we can carry out a timely study through non-invasive imaging studies, in order to initiate medical and surgical treatment according to the case and to identify, avoid or minimize the secondary complications or morbidity that it generates.
\end{abstract}

Keywords: cerebral venous thrombosis, acute suppurative otitis media, bacterial meningoencephalitis
Volume 10 Issue I - 2020

Ivan Cadena Vélez, Manuela Grego, Luis Siopa Internal Medicine Department, Hospital Distrital de Santarém, Portugal

Correspondence: Ivan Cadena Vélez, Internal Medicine Department, Hospital Distrital de Santarém, Portugal,Tel +579|4040788, Email icadenavele@gmail.com

Received: January 18, 2020 | Published: January 29, 2020

\section{Introduction}

Cerebral venous and sinus thrombosis (CVT) is an uncommon disease and accounts for $<1 \%$ of all strokes ${ }^{1,2}$ Multiple predisposing factors have been associated with CVT, among which only a few are reversible. Some of the transient conditions associated with CVT include pregnancy, dehydration, and infection. ${ }^{1}$ Cerebral venous thrombosis varies widely in its presentation, predisposition, neuroimaging, outcomes, and prognosis. ${ }^{3}$ The exact incidence and prevalence of CVT are unknown due to a lack of population based data. Although no age is exempt from cerebral venous thrombosis, in the pediatric population, neonates are most affected, and in adults, the highest incidence is in the third decade, with female preponderance. ${ }^{2}$

Thrombosis of cerebral venous sines (CVT) is considered difficult to diagnose due to the wide variety of signs and symptoms that can simulate a large number of other entities ${ }^{4,5}$ must be diagnosed as early as possible because it is potentially reversible and so that the specific medical treatment the surgeon can be quickly established due to the high morbidity that generates. ${ }^{4,6,7}$

\section{Case report}

A 65-year-old man with a history of ischemic heart disease with STEMI in 2018 and COPD, moderate ethanolism, and smoking admitted to the Emergency Department for four-day follow-up, fever, dyspnea, and temporary disorientation. worsened in the previous 24 hours with an altered state of consciousness. He was feverish $\left(39.4^{\circ} \mathrm{C}\right)$, sleepy, disoriented in space, and speech. The neurological examination found the reaction to painful stimuli, neck stiffness, positive Kernig sign, muscle strength $3 / 5$, and negative Babinski sign. Analytically stood out: Hb 13.4/dl,195x103/ $\mu \mathrm{L}, 16000 \times 108 /$ $\mu \mathrm{L}, 89.1 \%$, PCR $21.16 \mathrm{mg} / \mathrm{dl}$, glucose $188 \mathrm{mg} / \mathrm{dl}$, CK455U/L, lactate $2.5 \mathrm{mmol} / \mathrm{m}$ on blood gas analysis. CT-EC showed discrete collections of isohyperdense deposited in the occipital horns of the lateral ventricles, associated with an enlarged supra-tentorial ventricular system, with bulging contours and with a slight hypodensity in its surroundings, suggesting transpedicular edema, corresponding. A possible ventriculitis and a defect in the filling of the transverse sinus and left sigmoid in apparent relationship with thrombosis; and opacification of the middle ear by inflammatory process / otitis media. (Figure 1)
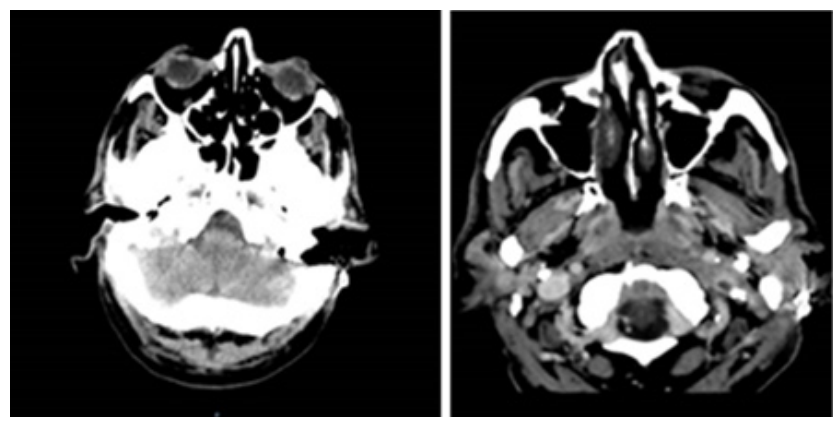

Figure I Otitis media. 
The study continued with a lumbar puncture, with cloudy CSF output. Cytochemical examination showed: 1353.9/dl proteins, glucose $<5 \mathrm{mg} / \mathrm{dl}, \mathrm{Cl}-113.2 / 1, \mathrm{LDH} 247 \mathrm{U} / \mathrm{L}, 8001 \mathrm{euc} / \mathrm{mm}^{3}$ with predominance of polymorphonuclear cells and some Gram-positive diplococci. Given these data, he was admitted to the intensive care unit for bacterial meningoencephalitis with a probable starting point for chronic left suppurative otitis media. He started antibiotic therapy with vancomycin $1 \mathrm{~g}$ and ceftriaxone $2 \mathrm{~g} 12 / 12 \mathrm{~h}$, plus dexamethasone $0.15 \mathrm{mg} / \mathrm{kg}(10 \mathrm{mg}) 6 / 6 \mathrm{~h}$. Ear-CT showed significant otomastoid filling on the left, with bone demineralization and potential communication with the endocranial space. (Figure 2)

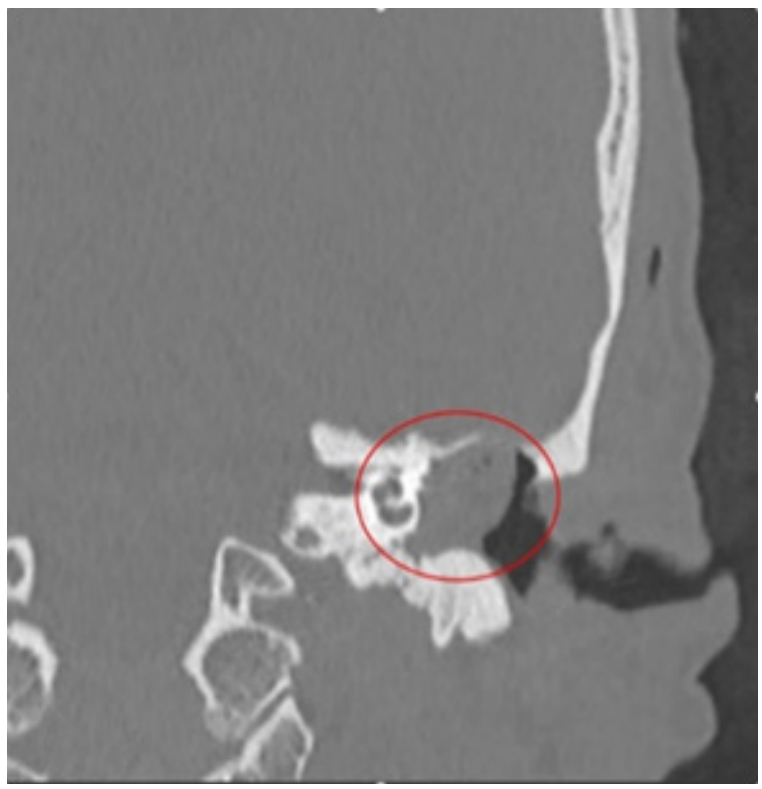

Figure 2 Endocranial space.

Due to hemodynamic instability, he was transferred to the ICU. He was underwent left tympanomastoidectomy, which showed dehiscence of the lateral sinus and gulf of the jugular, pus from the gulf of the jugular at the tip of the mastoid, and chronic cholesteatomatous otitis media, with an ongoing infectious process. In the CT-EC control, no de novo endocranial lesions were observed, and there was an apparent relationship between the sinus filling defect with thrombosis. (Figure 3)
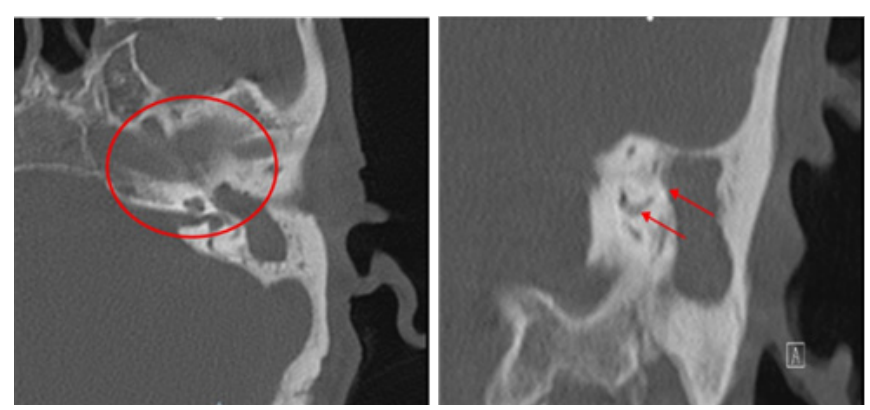

Figure 3 Sinus filling defect with thrombosis.

Blood cultures isolated $S$. pneumonia, sensitive to the antibiotic regimen instituted. Due to changes in hemodynamic and neurological status in the context of a possible recent subdural abscess, he was transferred to the neurosurgery service and died on the three-day postdrainage abscess in the neurological critical care unit.

\section{Discussion}

The CVT is a rare and difficult-to-diagnose pathology, so it is important to identify the underlying cause as part of the risk assessment and stratification of these patients, even if in up to a quarter of the cases, the risk is not identified cause. ${ }^{3,7}$ Local and systemic factors have been described as prevalent for CVT, but it should be taken into account that only the minority of cases involve, local factors, and even many patients exposed to these factors do not suffer from $\mathrm{CVT}^{7-9}$ include trauma, craniotomy, infection, arteriovenous malformation, central venous catheterization, and malignancy. The location of the CVT is partially determined by the causal mechanism; for example, patients with thrombosis of the cavernous sinus may present with sinusitis or infections of the face or orbit. ${ }^{9}$

In the same episode, the CVT commonly involves several venous sinuses presenting in the lateral sinuses in $64 \% 52 \%$ in the superior sagittal sinus in the sigmoid sinus $31 \%$, straight sinus $8 \%$ galen vein $6 \%$ and cavernous sinus $4 \%$. The sigmoid and transverse sinus thrombosis can originate as a complication of otitis media or mastoiditisdue to venous dissemination of the infection by the mastoid emissary vein or direct dissemination of the infection, as we assume happened to the patient already describedand it can also originate from the formation of extradural abscess that occurs in more than half of the cases. ${ }^{9,10-13}$

The symptoms that are observed most frequently in septic CVT are fever $88 \%$, otalgia $77 \%$, otorrhea $55 \%$, paresis of the abducens, papilledema 44\%; They can also attend with nuchal stiffness, edema, and periauricular erythema. ${ }^{10}$ Otoscopy is essential, especially if neurological symptoms are more prominent because, in these cases, the diagnosis can be more difficult. The osteogenic cause of lateral sinus thrombosis in children is otitis media and otitis media with effusion or mastoiditis, while in adults, the most common cause is cholesteatoma. Secondary to the septic CVT can cause cerebral infarction. The mechanisms of venous infarction are obstruction of the outflow, venous congestion, and a consequent increase in hydrostatic capillary pressure, extravasation of fluid into the interstitium, and the production of edema; $50 \%$ of cases of septic CVT progress to heart attack. $^{14-16}$ The infarction is produced by spreading the thrombus to the cortical veins causing vasogenic edema and focal areas of edema and cerebral hemorrhage. The signs of poor prognosis are hemorrhage on admission CT, deep venous thrombosis, and CNS infection; others described are male gender, altered state of consciousness, age over 40 years, Glasgow under 9, and deep cerebral venous thrombosis. ${ }^{17,18}$

The basis of treatment is the treatment of the underlying infection and the systemic stabilization of the patient. The initial choice of intravenous antibiotics should be made, taking into account the most common organisms involved in the site of suspicious origin of septic CVT. A reasonable initial combination may include ceftriaxone, vancomycin, and metronidazole to cover the broadest spectrum. ${ }^{14,19}$ Blood cultures and CSF should direct specific therapy. For toxic patients, the dose of ceftriaxone should be $2 \mathrm{~g}$ intravenously every 12 hours, vancomycin 750-1000mg intravenously every 12 hours, and metronidazole $7.5 \mathrm{mg} / \mathrm{kg}$ intravenously every 6 hours. Another suggested regimen is a combination of a third-generation cephalosporin, nafcillin, and metronidazole. ${ }^{20}$ The total duration of therapy is not clearly established and should depend on the clinical response, the primary site of infection and the associated complications, but a minimum of 3 to 4 weeks of intravenous therapy is required. ${ }^{14,21}$ The role of steroids is not well established in the 
treatment of septic CVT. They can help decrease inflammation and swelling, although this use is not well supported by the evidence.

Although it is standard for care in cases of aseptic CVT, the role of anticoagulants in the treatment of septic CVT is controversial. The theoretical advantage of anticoagulant therapy is the prevention of thrombus spread, as well as the possible inhibition of platelet function and anti-inflammatory effect through various mechanisms. The potential risk is intracranial and systemic hemorrhage. Another argument against the use of anticoagulants has been the assumption that the thrombus limits the infection and, therefore, prevents the spread of the infection. Anticoagulation may allow a greater extension of the infectious process. However, the reported incidence of bleeding in the context of septic CVT and the use of anticoagulants is extremely low. It has not been convincingly demonstrated that the mortality associated with septic CVT is reduced with the combined use of antibiotics and anticoagulants, although there was a tendency towards a decrease in morbidity when anticoagulants were used early together with antibiotics. Currently, available evidence favors the use of anticoagulants together with antibiotics early in the course of septic CVT; however, clear protocols or target anticoagulation values have not been established. Intravenous heparin should be used initially, followed by oral anticoagulants. The optimal duration of treatment is unknown.

Surgical drainage of the cavernous sinus is almost never performed. Surgical treatment is reserved for the primary site of infection, which may require drainage of the primary focus, particularly if the focus is on a non-draining site. ${ }^{1}$

In the pre-antibiotic era, septic CVT was almost always fatal. Despite all the advances in diagnosis and treatment, mortality rates are still reported at around $30 \%$, with significant morbidity in survivors. ${ }^{1}$ Residual deficits in survivors include partial or complete visual loss, cranial nerve abnormalities, pituitary dysfunction, seizures, hemiparesis, facial disfigurement, as well as personality changes. Full recovery is achieved in less than $50 \%$ of patients.

\section{Conclusion}

The CVT is an infrequent pathology, considered difficult to diagnose due to the wide variety of signs and symptoms, being able to simulate a large number of other entities. ${ }^{4,5}$ And even more so if the trigger is a non-systemic pathology, especially of septic origin. It is important to have this diagnosis always present, and it is essential that after the diagnostic suspicion we can carry out a timely study through non-invasive imaging studies, in order to initiate medical and surgical treatment according to the case and to identify, avoid or minimize the secondary complications or morbidity that it generates..$^{5-7}$ It is worth mentioning that adequate anamnesis and a complete physical examination are essential to achieve diagnoses such as venous sinus thrombosis caused by parameningeal infections whose incidence is very low.

\section{Acknowledgments}

None.

\section{Conflicts of interest}

The authors declare that they have no conflicts of interest regarding this article.

\section{Sources of funding}

The authors declare that they have not received subsidies or scholarships for the elaboration of the article.

\section{References}

1. Saposnik G, Barinagarrementeria F, Brown RD, et al. Diagnosis and management of cerebral venous thrombosis: a statement for healthcare professionals from the American Heart Association/American Stroke Association. Stroke. 2011;42(4):1158-1192.

2. Einhäupl K, Stam J, Bousser MG, etal. European Federation of Neurological Societies. EFNS guideline on the treatment of cerebral venous and sinus thrombosis in adult patients. Eur J Neurol. 2010;17(10):1229-1135.

3. Wasay M, Bakshi R, Bobustuc G, et al. Cerebral venous thrombosis: analysis of a multicenter cohort from the United States. J Stroke Cerebrovasc Dis. 2008;17(2):49-54.

4. Fischer C, Goldstein J, Edlow J. Cerebral venous sinus thrombosis in the emergency department: Retrospective analysis of 17 cases and review of the literature. J Emerg Med. 2010;38(2):140-147.

5. Bushnell C, Saposnik G. Evaluation and management of cerebral venous thrombosis. Continuum (Minneap Minn). 2014;20:335-351.

6. Rodallec M, Krainik A, Feydy A, et al. Cerebral venous thrombosis and multidetector ct angiography: Tips and tricks. Radiographics. 2006;26:S5-18.

7. Leach JL, Fortuna RB, Jones BV, et al. Imaging of cerebral venous thrombosis: Current techniques, spectrum of findings, and diagnostic pitfalls. Radiographics. 2006;26:S19-43.

8. Barinagarrementeria F, Arauz A. Evaluation and management of a patient with possible cerebral venous thrombosis. Neurol Clin Pract. 2014;4(5):394-401.

9. Mc Bane RD, TafurA, Wysokinski W. Acquired and congenital risk factors associated with cerebral venous sinusthrombosis. Thrombs Res. 2010;126(2):81-87.

10. Iwashita T, Kitazawa K, Koyama JI, et al. Subtle computed tomography abnormalities in cerebral deep sinus thrombosis. J Clin Neurosci. 2007;14(1):68-71

11. Ropposch T, Nemetz U, Braun EM, et al. Low molecular weight heparin therapy in pediatric otogenic sigmoid sinus thrombosis: A safe treatment option? Int JPediatr Otorhinolaryngol. 2012;76(7):1023-1026.

12. Unsal EE, Ensari S, ko C. A rare and serious complication of chronic otitis media: Lateral sinus thrombosis. Auris Nasus Larynx. 2003;30(3):279282

13. Southwick FS, Richardson EP Jr, Swartz MN. Septic thrombosis of the dural venous sinuses. Medicine (Baltimore).1986;65(2):82-106.

14. Ebright JR, Pace MT, Niazi AF. Septic thrombosis of the cavernous sinuses. Arch Intern Med. 2001;161(22):2671-2676.

15. Bhatia K, Jones NS. Septic cavernous sinus thrombosis secondary to sinusitis: are anticoagulants indicated? A review of the literature. $J$ Laryngol Otol. 2002;116(9):667-676.

16. Dlamini N, Billinghurst L, Kirkham F. Cerebral venoussinus (sinovenous) thrombosis in children. Neurosurg Clin N Am. 2010;21(3):511-527.

17. Koopman K, Uyttenboogaart M, Vroomen PC, et al. Development and validation of a predictive outcome score of cerebral venous thrombosis. $J$ Neurolo Sci. 2009;276(1-2):66-68.

18. Kojan S, Al-Jumah M. Infection related cerebral venous thrombosis. $J$ Pak Med Assoc. 2006;56(11):494-497. 
19. Desa V, Green R. Cavernous sinus thrombosis: current therapy. J Oral Maxillofac Surg. 2012;70(9):2085-2091.

20. DiNubile MJ. Septic thrombosis of the cavernous sinuses. Arch Neurol. 1988;45(5):567-572.
21. Levine SR, Twyman RE, Gilman S. The role of anticoagulation in cavernous sinus thrombosis. Neurology. 1988;38(4):517-522. 